\title{
Application of Femtosecond Laser Peen Forming to Sheet Metal Bending
}

\author{
Yoshihiro Sagisaka ${ }^{*}$, Masayoshi Kamiya ${ }^{* *}$, Minoru Matsuda ${ }^{* * *}$ and Yukihiro Ohta ${ }^{* * * *}$ \\ *Industrial Research Institute of Shizuoka Prefecture, \\ 2078 Makigaya, Aoi-ku, Shizuoka, Shizuoka 421-1298, Japan \\ E-mail: sagisaka@iri.pref.shizuoka.jp \\ ** Fuji Technical Support Center, Industrial Research Institute of Shizuoka Prefecture, \\ 2590-1 Ohbuchi, Fuji, Shizuoka 417-8550, Japan \\ *** The Shizuoka Organization for Creation of Industries, \\ 44-1 Otemachi, Aoi-ku, Shizuoka, Shizuoka 420-0853, Japan \\ **** Hamamatsu Technical Support Center, Industrial Research Institute of Shizuoka Prefecture, \\ 1-3-3 Shin-miyakoda, Kita-ku, Hamamatsu, Shizuoka 431-2103, Japan
}

\begin{abstract}
A focused femtosecond laser can induce shock waves at irradiated target surface. Under some irradiation conditions, the shock waves are powerful enough to deform a metal target plastically. Laser peen forming is a sheet metal forming process using this deformation. The author adopted laser peen forming with femtosecond laser as a thin-sheet-metal bending. This process is appropriate for small parts production. The forming properties of a pure aluminum sheet were investigated. Some irradiation conditions, fluence and density of irradiated pulses, were changed. Using clarified forming properties, some pieces of phosphor bronze and stainless steel sheets were bent in various shapes. Some of these bent shapes are difficult to achieve with conventional press forming. Simple change of processing parameters and scanning paths allowed to form various shapes. This result means that the process is suitable for multikind small lot production.

DOI:10.2961/jlmn.2012.02.0006
\end{abstract}

Keywords: laser peen forming, femtosecond laser, bending, thin-sheet-metal, laser induced shock wave, forming property

\section{Introduction}

A focused short-pulse laser can induce shock waves at a target surface. The shock waves can deform a metal target plastically under some irradiation conditions [1]. Laser peening [2-3] is an application of such deformations. The compressive residual stress, which is induced by the deformations, extends the service life of irradiated part. Vollertsen has reported a non-thermal laser shock forming based on laser induced shock waves [4]. A stretch-forming of thin-sheet-metal was performed with excimer laser and with TEA- $\mathrm{CO}_{2}$-Laser. Another application for forming is laser peen forming. It is a die-less and non-contact sheet metal forming method using the deformations. Some aircraft skin panels have already been formed by laser peen forming with a nanosecond laser [5-6]. Laser forming is another well known die-less non-contact sheet metal forming method using laser [7]. It is based on a thermal effect generated by laser irradiation. Contrary to laser forming, laser peen forming is based on a mechanical effect. Therefore, they have entirely different forming properties.

A femtosecond laser is a type of ultrashort-pulse laser. A focused femtosecond laser, also, can induce shock waves at a target surface. The pulse energy of femtosecond laser is usually much lower than that of nanosecond laser. Therefore, the shock waves induced by femtosecond laser are too small to form thick plates. However, they are powerful enough to form thin-sheet-metals. Thin-sheet-metals are suitable materials for micro parts. The authors attempted to apply the deformations induced by femtosecond laser irradiation to micro parts productions. The laser peen forming with femtosecond laser was adopted as a thin-sheet-metal bending [1]. The forming properties of this process depend on the irradiation conditions and scanning parameters. In order to investigate the forming properties, some aluminum sheets were bent with various irradiation conditions and scanning parameters. In addition, as the applications of clarified forming properties, several thin-sheet-metals used for springs were bent into various shapes.

\section{Principle of laser peen forming}

When a high intensity short laser pulse is focused on a sheet metal, the irradiated top surface is ionized and removed instantaneously, and a high-pressure plasma is generated (laser ablation). Although the plasma expands rapidly, the inertial force of atmosphere confines the plasma expansion. As a result, an impulse is transferred to the sheet metal. The irradiated surface is subjected to a high-pressure and a shock wave propagates into the sheet metal. The

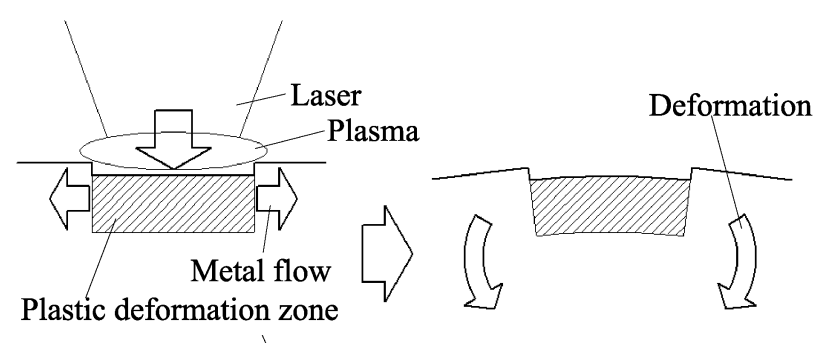

Sheet metal
(a) Irradiation
(b) Deformation

Fig. 1 Principle of laser peen forming 
surface is pressed and elongated to the lateral direction plastically, and the sheet metal is bent as shown in Figure 1. Laser peen forming is achieved by scanning the laser over whole surface and accumulating such deformations.

A similar sheet metal forming process, which is called peen forming, has already been developed [8]. It is an application of shot peening and uses deformations generated by impacts of shots thrown to a sheet metal. The deformation mode of peen forming is changed by the depth of plastic deformation zone [9], as shown in Figure 2. When the deformation zone is small, only the peened surface is elongated (mode I). The sheet metal is curved as peened surface is convex. When the deformation zone reaches to the bottom, the behind face starts to elongate and the curvature decreases (mode II). With the growth of the deformation zone, the elongation of behind face reaches that of peened face (mode III). The sheet metal is kept flat by this mode. When the deformation zone grows larger, the elongation of behind face exceeds that of peened face at last (mode IV). The sheet metal is curved as peend face is concave. The depth of deformation zone relates to peening energy and sheet material. A high energy and a soft material enlarge the plastic deformation zone. In addition, the deformation modes are decided by the relative depth of plastic deformation zone to sheet thickness. The sheet thickness, also, relates to the deformation modes.

Laser peen forming uses laser induced shock waves instead of the shot impacts. Therefore, these two processes have similar forming properties. In the case of laser peen forming, the depth of deformation zone depends on the magnitude of shock wave. The pressure of shock wave correlates with the intensity of laser [10]. The intensity can replace the peening energy of Figure 2 . When the intensity is low or sheet thickness is large, the deformation mode $\mathrm{I}$ is obtained. When the intensity is high or thickness is small, the deformation became mode IV. Hu et al. have confirmed these changes of deformation mode according to intensity and sheet thickness [11].

A nanosecond laser can induce strong shock waves. However, it needs high pulse energy to induce shock waves. It is difficult for nanosecond laser to induce weak shock waves with low energy. When a thin-sheet-metal is formed

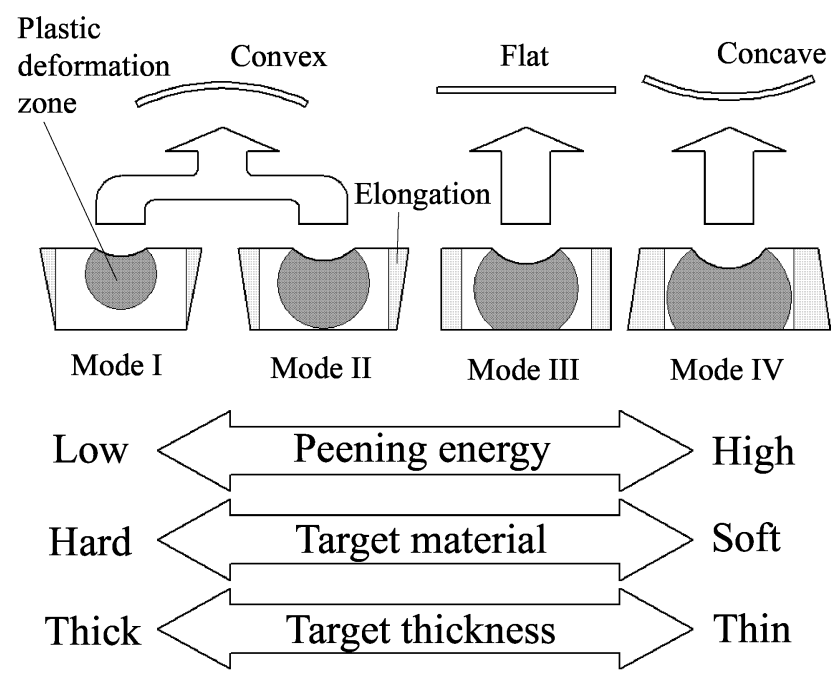

Fig. 2 Deformation modes of peen forming by such strong shock waves, the deformation mode usually becomes mode IV [12-13]. This direction of curve is same as that obtained by usual laser forming. It is not so valuable deformation. In order to obtain convex curve, the laser must be irradiated on another thin metal foil pasted on the sheet metal surface [14]. The foil acts as a shock absorber, and weakened shock waves can generate deformation mode I on the sheet metal.

In contrast, a femtosecond laser can induce weak shock waves with low pulse energy. Such shock waves are appropriate to curve a thin-sheet-metal as irradiated surface is convex. Laser peen forming with nanosecond laser sometimes needs a coating on the sheet, in order to protect the sheet from thermal effect [12]. Femtosecond laser induces very little thermal effect. This property is appropriate for small parts which hate thermal affected zone. In addition, laser peen forming with nanosecond laser sometimes needs water film on the sheet $[11,15]$. The water confines the plasma expansion strongly and increases shock waves. However, in the case of femtosecond laser, the water does not always contribute to the deformation. Because, the water absorbs focused femtosecond laser pulses and decrease its energy easily [16]. The coating and water film are not necessary for femtosecond laser peen forming. These properties contribute to simplify the processing set-up.

\section{Experimental equipment and procedure}

The femtosecond laser used in this study was Ti:sapphire laser amplified by chirped pulse amplification. The wavelength was about $800 \mathrm{~nm}$; the pulse duration was about $80 \mathrm{fs}$. The pulse frequency was variable from $1 \mathrm{~Hz}$ to $1 \mathrm{kHz}$. The summary of equipment is shown in Figure 3. The pulse energy $E$ was controlled by a neutral density filter (ND filter). $E$ was $200 \mu \mathrm{J}$ in maximum. The laser beam was focused by a focal lens which has a focal length $f=35 \mathrm{~mm}$. The surface of the workpiece was monitored by a CCD camera provided with a long-distance microscopic

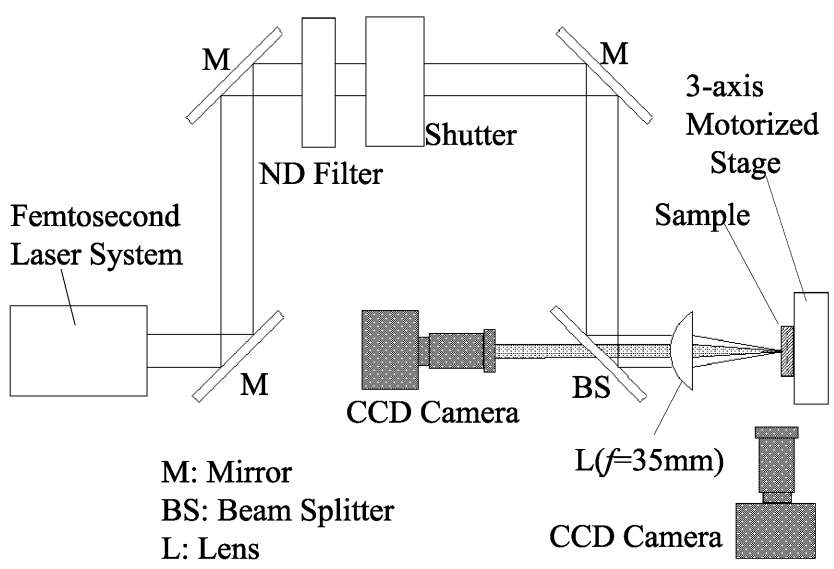

Fig.3 Schematic diagram of experimental equipment

Table 1 Materials used for experiments

\begin{tabular}{ccc}
\hline & Thickness $t$ & Vickers hardness Hv \\
\hline Pure aluminum & $0.10 \mathrm{~mm}$ & 50.0 \\
Phosphor bronze & $0.05 \mathrm{~mm}$ & 240 \\
Stainless steel & $0.05 \mathrm{~mm}$ & 435 \\
\hline
\end{tabular}


lens. Using the monitored image, the workpiece was moved to the desired position.

Austenitic stainless steel (SUS304 in JIS, S30400 in ASTM), phosphor bronze (C5191P in JIS) and pure aluminum (A1100 in JIS, 1100 in ASTM) were adopted as workpieces. Thickness $t$ and Vickers hardness of each material are shown in Table 1. Stainless steel and phosphor bronze are typical materials used for springs. Pure aluminum was selected in order to investigate forming properties, because it had a better reproducibility than other materials.

The outline of the experimental procedure is shown in Figure 4. Materials were sheared into stripes of $30 \mathrm{~mm}$ length and $5 \mathrm{~mm}$ width. A workpiece was mounted on a 3axis motorized stage with clamps. The clamped workpiece was scanned over the hatched area of circle 'A'. The length of scanning area $w$ was changed according to the shape of the goal. In the case of forming property investigation, $4 \mathrm{~mm}$ was used for $w$. Laser scanning was performed by moving the motorized stage. Scanning speed was $5 \mathrm{~mm} / \mathrm{s}$. Left of Figure 4 shows the scanning path. Line scanning was repeated several times. The lateral scanning pitch $p_{l}$ was decided by used pulse frequency [16]. The workpiece was shifted toward longitudinal direction after every line scanning. The longitudinal scanning pitch $p_{2}$ was changed by scanning program. A spot diameter $d$ was controlled by shifting the workpiece axial to the direction of the laser beam. The focal point was defined as the original point of defocus distance $z$. When the workpiece was shifted toward the beam source, $z$ was defined positive, as shown in Figure 5. The relationship between $z$ and spot diameter $d$ is shown in Figure 6. The diameter of ablation mark left on an aluminum target was defined as $d$. After the scanning, the workpieces were released from the clamps and bending angles $\theta$ were measured.



DETAIL A

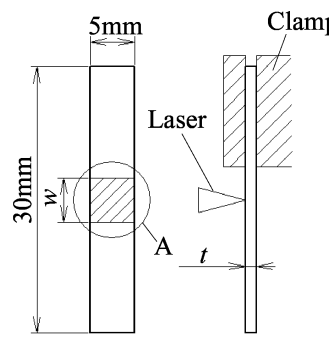

(a) During irradiation

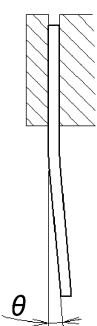

(b) After irradiation
Fig.4 Schematic diagram of experimental set-up

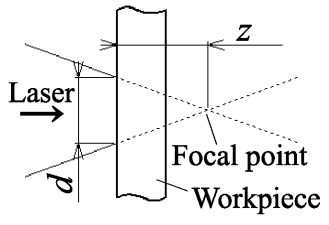

(a) $z>0$

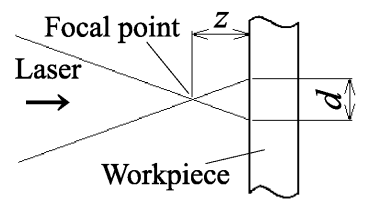

(b) $z<0$
Fig.5 Definition of defocus distance

\section{Experimental Results}

\subsection{Fundamental forming property}

Three materials were bent in about 10 degrees, as shown in Figure 7. The workpiece centre, which has dull surface, is scanned area. The deformation generated by a single pulse, which is illustrated in Figure 1, is axial symmetrical with respect to the beam axis. Therefore, the curvature to the width direction, also, occurred. When the bending angle was very small, the scanned area was formed as dome. However, when the bending angle increased, the curvature to longitudinal direction became superior and the curvature to the width direction disappeared. It was because non-irradiated areas, which were adjacent to the scanned area, restricted the curvature to the width direction. The curvature to the width direction was difficult to find in the workpieces shown in Figure 7.

In the case of laser forming, a thermal conductivity influences the bending efficiency very much. Stainless steel, which has low thermal conductivity, is easy to bend, but pure aluminum, which has low conductivity, is difficult to bend. In contrast, in the case of laser peen forming, the stainless steel needed the same pulse energy and pulse frequency as the pure aluminum, in spite of thinner thickness. Soft material was easy to bend. The bending efficiency related to the flow stress. This fact shows that laser peen forming is based on mechanical effects.

Very large bending radii were obtained in Figure 7. It is difficult for conventional press forming to keep precise bending angles with such large bending radii. It is because such thin-sheet-metal bending is accompanied by large and unstable spring back phenomena. Laser peen forming is a kind of incremental forming. The bending deformation is an accumulation of a lot of small deformations and appears incrementally. In addition, there is no die which causes elastic deformation to the workpiece during the process. The spring back as press forming never occurs essentially. This forming property is useful to form spring materials which have excessively large spring back.

Applications of such forming property are shown in Figure 8. Figure 8(a) is a phosphor bronze bent into a wave shape. It was formed by scanning both sides alternately. Several constant large bending radii were formed. Figure $8(\mathrm{~b})$ is a phosphor bronze scanned diagonally. The workpiece was bent at right angles to line scanning direction. In order to reduce the error caused by spring back, applying

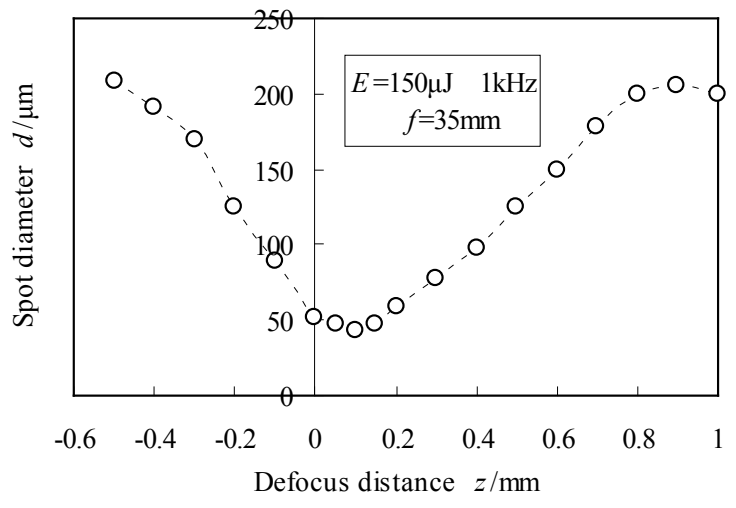

Fig.6 Relationship between defocus distance and spot diameter 


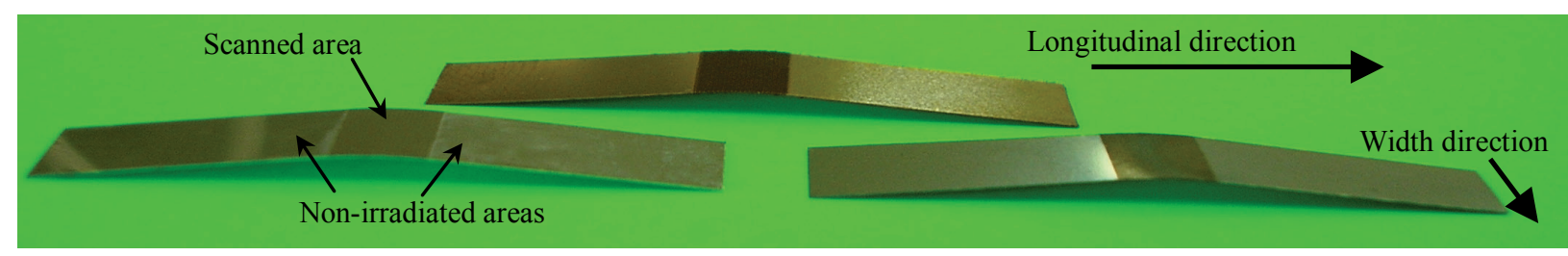

Fig.7 Examples of bent three materials

Left: Pure aluminum ; $500 \mathrm{~Hz}, p_{2}=100 \mu \mathrm{m}, E=200 \mu \mathrm{J}, z=0.1 \mathrm{~mm}$

Center: Phosphor bronze ; $500 \mathrm{~Hz}, p_{2}=100 \mu \mathrm{m}, E=150 \mu \mathrm{J}, z=-0.1 \mathrm{~mm}$

Right: Stainless steel ; $500 \mathrm{~Hz}, p=100 \mu \mathrm{m}, E=200 \mu \mathrm{J}, z=0.1 \mathrm{~mm}$

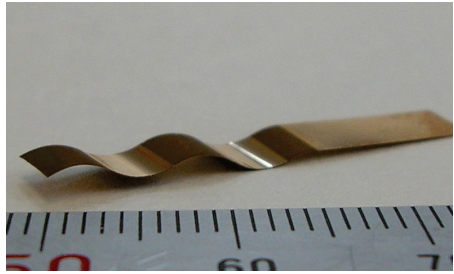

(a) Wave shape

$\left(1 \mathrm{kHz}, p_{2}=50 \mu \mathrm{m}\right.$, $E=150 \mu \mathrm{J}, z=0.1 \mathrm{~mm}$ ) $E=150 \mu \mathrm{J}, z=0.2 \mathrm{~mm})$

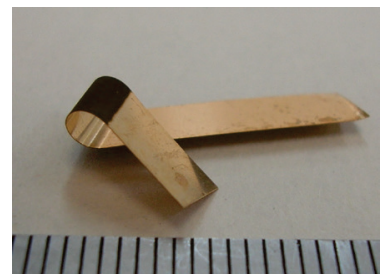

(b) Spiral shape

$\left(1 \mathrm{kHz}, p_{2}=25 \mu \mathrm{m}\right.$,

Fig.8 Phosphor bronze bent into mild curvature

an excessive bending is usual for press forming. However, excessive bending is inapplicable to such shape, because the workpiece interferes with itself. These shapes are not so easy for other processes, which are accompanied by spring back, to achieve.

\subsection{Effect of fluence}

The pulse duration was constant in this study; therefore intensity was in proportion to fluence $F$. Therefore, the magnitude of shock wave related to $F$ in this paper. The effects of fluence on bending angles were investigated. Pure aluminum workpieces were bent with various pulse energies $E$ and defocus distances $z$. The scanning pitches $p_{2}$ and $p_{1}$, namely pulse frequency, were constant. The length of scanning area $w$ was $4 \mathrm{~mm}$ constant. The relationships between $z$ and $\theta$ are shown in Figure 9. According to Figure $6, \theta$ became maximum when $d$ was minimum. And $\theta$ increased with $E$. The relationship between $F$ and $\theta$ is shown in Figure 10. $\theta$ increased almost proportionally to the logarithm of $F$. High fluence was favorable for obtaining small bending radius. When the deformation mode II appears, $\theta$ must decrease with the fluence. However, such behavior was not observed. Obtained deformations were those of mode I. Therefore, the depth of plastic deformation zone obtained by femtosecond laser irradiation was smaller than the sheet thickness.

These results indicate that the bending radius is changeable by changing the fluence, namely $E$ or $z$. When $z$ was changed gradually during the scanning, the bending radius was changed continuously. An example is shown in Figure 11. The scanning was started from an end. The $z$ was enlarged from 0.2 to $1 \mathrm{~mm}$ during the scanning. The bending radius was small at the end of workpiece, which was irradiated with small spots. However, the bending radius became large with the increase of spot diameter.

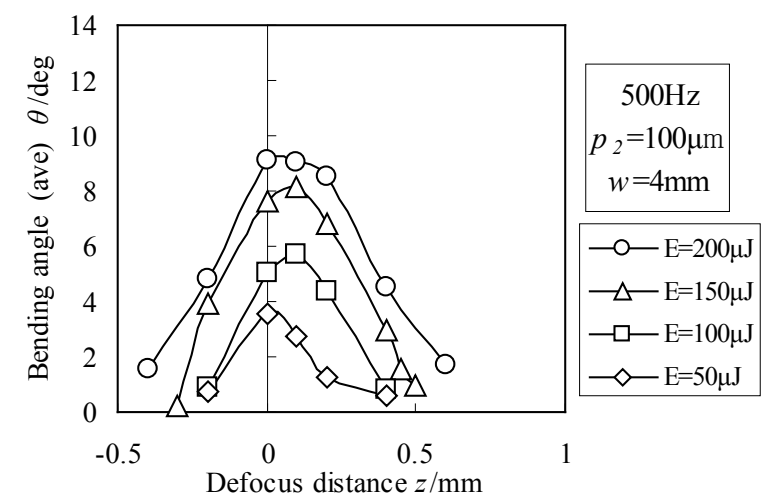

Fig.9 Relationship between defocus distance and bending angle

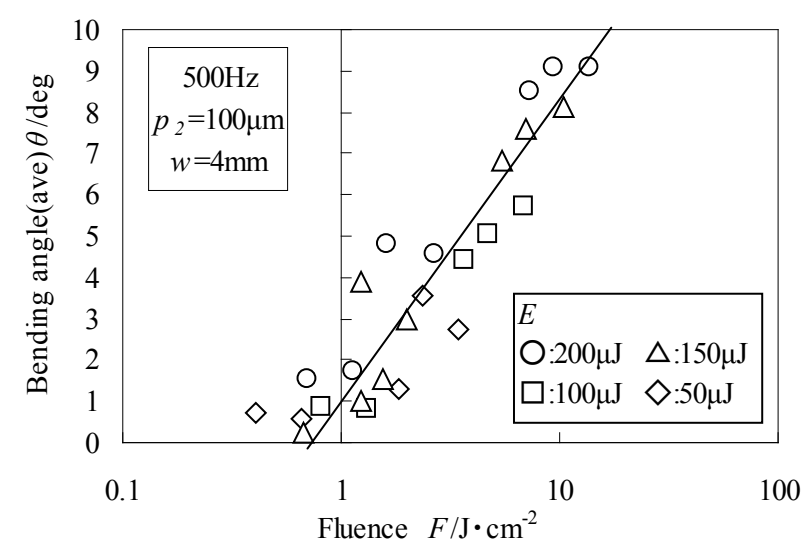

Fig.10 Relationship between fluence and bending angle

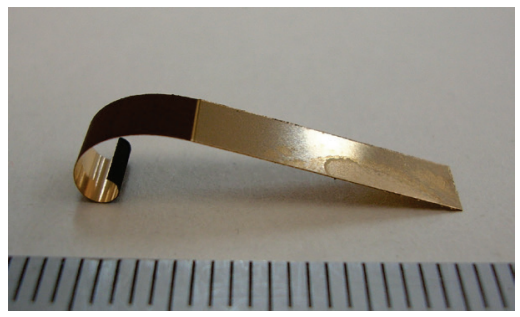

Fig.11 Scroll shape using effect of fluence (phosphor bronze) $1 \mathrm{kHz}, p_{2}=10 \mu \mathrm{m}, E=150 \mu \mathrm{J}, z=0.2 \rightarrow 1 \mathrm{~mm}$ 


\subsection{Effect of irradiated pulse density}

Pure aluminum workpieces were bent with various irradiated pulse densities $D_{i p}$. The longitudinal scanning pitch $p_{2}$ was $100 \mu \mathrm{m}$ constant. $D_{i p}$ was controlled by changing $p_{1}$, namely pulse frequency. When the frequency was $1 \mathrm{kHz}, D_{i p}$ was maximum 2000pulses $/ \mathrm{mm}^{2}$. The length of scanning area $w$ was $4 \mathrm{~mm}$ constant. The change of bending angles $\theta$ with $D_{i p}$ is shown in Figure 12. $\theta$ increased with $D_{i p}$. It means that increasing the irradiated pulse density allows small bending radius. The equipment used in this study was easy to change the pulse frequency and the scanning path. Changing the irradiated pulse density is one of useful methods to control the deformation.

When much smaller $p_{2}$ are used, much smaller bending radius is possible. The increase of fluence, also, decreases the bending radius. Examples of stainless steel workpieces, which were bent with higher fluence and different $p_{2}$, are shown in Figure 13. Phosphor bronze workpieces, which were bent with high fluence and high irradiated pulse density, are shown in Figure 14. An application of deformation control using $D_{i p}$ is shown in Figure 15. A workpiece was bent into different directions with different bending radii. Various bent shapes were achieved by changing pulse density, fluence and scanning paths. These workpieces showed that this process was favourable for multikind small lot production, for example trial production of some flat spring parts.

\subsection{Influence of laser stability}

The laser emitter needs periodic maintenance. Figure 13(b) and Figure 14(a) were processed with same irradiation condition but before and after a maintenance, respectively. Although a softer material is easy to bend, stainless

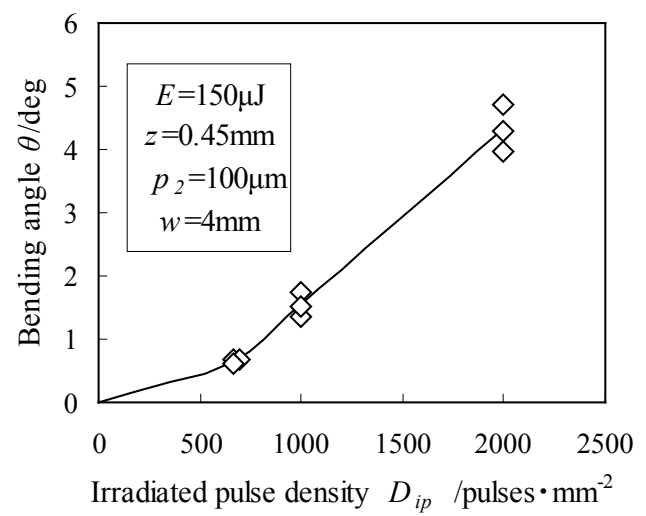

Fig.12 Change of bending angle with irradiated pulse density

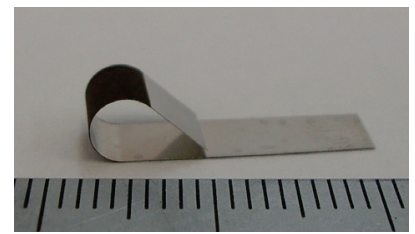

(a) $p_{2}=25 \mu \mathrm{m}$ $D_{i p}=8000$ pulses $/ \mathrm{mm}^{2}$

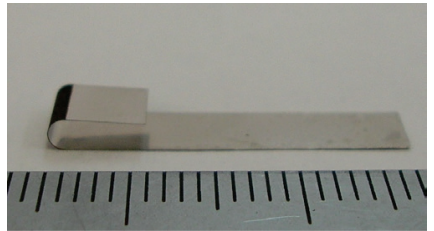

(b) $p_{2}=10 \mu \mathrm{m}$, $D_{i p}=20000$ pulses $/ \mathrm{mm}^{2}$
Fig.13 Effect of pulse density on stainless steel bending $(E=200 \mu \mathrm{J}, z=0.1 \mathrm{~mm})$ steel showed a little smaller bending radius than phosphor bronze. It was because there was remarkable decrease of bending efficiency after the maintenance, as shown in Figure 16. The phenomena enlarged the bending radii of Figure 14(a). The phenomena appeared in stainless steel and phosphor bronze, but appeared more remarkably in stainless steel which was harder material. In contrast, they appeared unclearly in pure aluminum. Therefore, they were not due to deformation mode II. And they appeared remarkably with higher fluence.

Changes of beam profile and spectrum distribution were observed after the maintenance. The spectrum distribution is somewhat controllable. Some experiments were conducted with various spectrum distributions, but the bending efficiency was kept constant. The change of beam profile was considered as the primary cause of the phenomena. The comparison of beam profile is shown in Figure 17. Interference patterns of Figure 17(a) were due to contaminations. The bending direction corresponded to vertical direction of the figures. The profile was corrected like Gaussian distribution by the maintenance. The change of fluence distribution, namely the beam profile, changes the shock wave pressure distribution in the spot and the strain distribution. When the profile has Gaussian distribution, metal flow is axial symmetrical with respect to the beam axis as a ball indentation. However, the vertically shortened distribution as Figure 17(a) shows metal flow

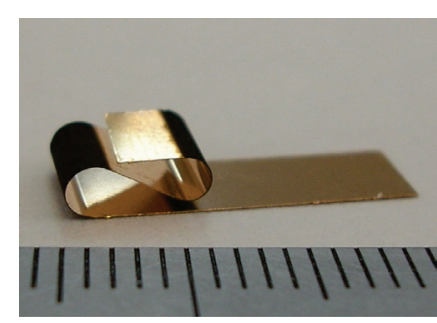

(a) S-bending

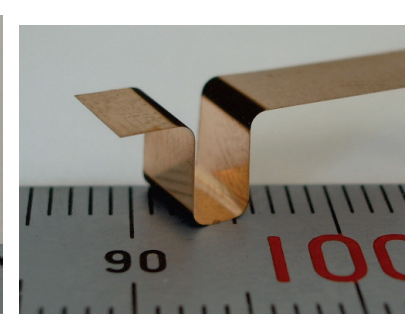

(b) Hat bending,
Fig.14 Examples of severe curvature $\left(D_{i p}=20000 \mathrm{pulses} / \mathrm{mm}^{2}\right.$, $E=200 \mu \mathrm{J}, z=0.1 \mathrm{~mm}$ )

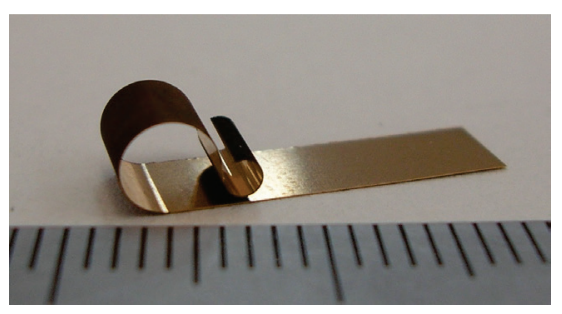

Fig.15 Bent phosphor bronze using different pulse densities $\left(D_{i p}\right.$ were 8000 and 20000 pulses $/ \mathrm{mm}^{2}, E=200 \mu \mathrm{J}$, $z=0.1 \mathrm{~mm})$

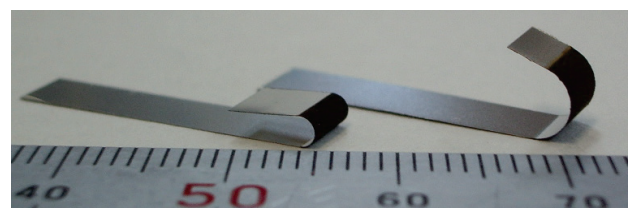

Fig.16 Influence of laser emitter maintenance on stainless steel (left: before maintenance right: after maintenance) $p_{2}=10 \mu \mathrm{m}, D_{i n}=20000$ pulses $/ \mathrm{mm}^{2}, E=200 \mu \mathrm{J}, z=0.1 \mathrm{~mm}$ 
somewhat like that of a roller indentation as Figure 18. Metal flow toward circumferential direction is dominant in the roller indentation. It was considered that the profile before the maintenance encouraged metal flow toward bending direction and was advantageous for the bending efficiency.

On the other hand, the phenomena appeared clearly in the materials which have low thermal conductivity. The effect of beam profile is not enough to explain such difference by materials. The femtosecond laser irradiation generates very small thermal effect but not nil. Some thermal effect is unavoidable with high fluence. The phenomena appeared clearly with higher fluence. It was considered that the thermal effect as laser forming acted. However, the mechanism of the phenomena is not yet specified. The change of the bending efficiency is serious problem for reproducibility. The laser stability is very important for the process.

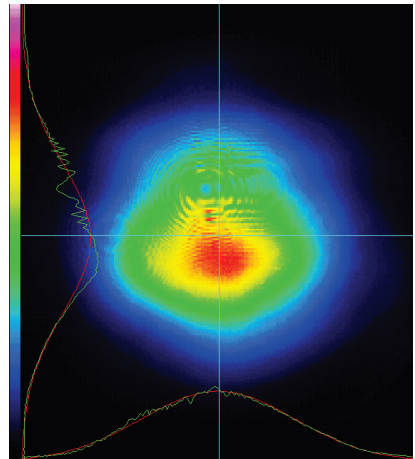

(a) Before maintenance

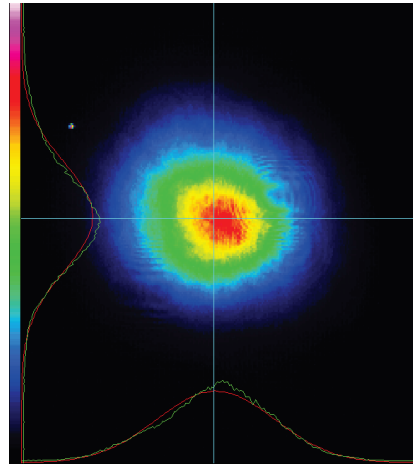

(b) After maintenance
Fig.17 Comparison of beam profile

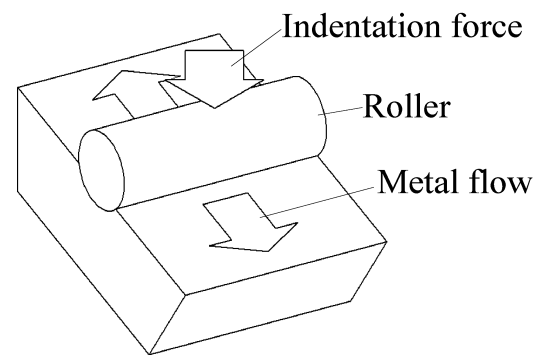

Fig.18 Roller indentation

\section{Summary}

Femtosecond laser peen forming was applied to thinsheet-metal bending, in order to utilize for small parts production. Thin-sheet-metals used for springs were bent to various shapes. Some of these shapes, for example large bending radii, were difficult for usual press forming to achieve because of large spring back. This process is free from spring back and favourable for the bending of spring materials. The effects of fluence and irradiated pulse density were investigated. High fluence and high irradiated pulse density decreased bending radius of sheets. These parameters enable to control the bending radius. Several complicated shapes were achieved by simple change of fluence, irradiated pulse density and scanning path. These unique forming properties are favourable for multikind small lot production. However, this process had troubles in reproducibility. High laser stability is the most important subject for a future study.

\section{Acknowledgment}

This work is a part of a project research program which is supported by Shizuoka prefecture.

\section{References}

[1] Y. Sagisaka: Proc. 9th Int. Conf. on Technol. of Plasticity, Gyeongju, (2008) p.1878.

[2] Y. Sano, T. Adachi, I. Altenberger, M.A. Cherif, B. Scholtes, K. Masaki, Y. Ochi, and T. Inoue: Key Eng. Mater., 345-346, (2007) 1589.

[3] R. Specht, F. Harris, L. Lane, D. Jones, L. Hackel, T. Zaleski, J. Halpin, M. Hill, and W. Wübbenhost: Proc. 8th Int. Conf. on Shot Peening, Munich, (2002) 2002062

[4] F. Vollertsen: Proc. 8th Int. Conf. on Technol. of Plasticity, Verona, (2005) p.557.

[5] Lawrence Livermore National Laboratory: Sci. Technol. Rev., 2003, (2003) 16.

[6] P. O'Hara: Proc. 8th Int. Conf. on Shot Peening, Munich, (2002) 2002030.

[7] Y. Namba: Proc. Int. Conf. Lasers '85, Las Vegas, (1986) p.403.

[8] D.L. Baughman: Proc. 2nd Int. Conf. on Shot Peening, Chicago, (1984) p.28.

[9] K. Kondo, S. Tsuzuki, and A. Kato: Proc. 1st Int. Conf. on Shot Peening, Paris, (1981) p.565.

[10] R. Fabbro, J. Fournier, P. Ballard, D. Devaux. and J. Virmont: J. Appl. Phys., 68(2), (1990) 775.

[11] Y. Hu, X. Xu, Z. Yao, and J. Hu: J. Appl. Phys., 108, (2010) 073117.

[12] K.R. Edwards, S.P.Edwardson, C. Carey, G. Dearden, and K.G. Watkins: Int. J. Adv. Manuf. Technol., 47, (2010) 191.

[13] J.L. Ocaña, M. Morales, J.A. Porro, O. García, J.J. García-Ballesteros, and C. Molpeceres: J. Laser Micro/ Nanoengineering, 4. (2009) 55.

[14] Y. Fan, Y. Wang, S. Vukelic. and Y.L. Yao: J. Appl. Phys., 98, (2005) 104904.

[15] M.Morales, J.A. Porro, J.J. García-Ballesteros, C. Molpeceres, and J.L. Ocaña: Appl. Surface Science, 257. (2011) 5408.

[16] Y. Sagisaka, M. Kamiya, M. Matsuda, and Y. Ohta: J. Mater. Processing Technol., 210, (2010) 2304.

(Received: December 01, 2011, Accepted: March 27, 2012) 